\title{
Rimegepant: first novel oral calcitonin gene-related peptide inhibitor for migraine
}

\author{
Saravana Kumar Ramasubbu ${ }^{1}$, Senkadhirdasan Dakshinamurthy², Sarika Palepu ${ }^{3}$, \\ Arkapal Bandyopadhyay ${ }^{1 *}$, Sathish Kumar Rajendran ${ }^{4}$
}

\author{
${ }^{1}$ Department of Pharmacology, ${ }^{2}$ Department of Community and Family medicine, All India Institute of Medical \\ Sciences, Rishikesh, Uttarakhand, India \\ ${ }^{3}$ Department of Community and Family medicine, Vir Chandra Singh Garhwali Government Institute of Medical \\ Sciences and Research, Srinagar, Uttarakhand, India \\ ${ }^{4}$ MBBS, Sri Muthukumaran Medical college hospital and Research institute, Chennai, Tamil Nadu, India
}

Received: 20 March 2020

Accepted: 10 April 2020

\section{*Correspondence:}

Dr. Arkapal Bandyopadhyay,

Email: drarkapal@gmail.com

Copyright: (c) the author(s), publisher and licensee Medip Academy. This is an open-access article distributed under the terms of the Creative Commons Attribution Non-Commercial License, which permits unrestricted non-commercial use, distribution, and reproduction in any medium, provided the original work is properly cited.

\begin{abstract}
Migraine is a neurological condition characterized by intense, debilitating headaches. Symptoms may include nausea, vomiting, numbness or tingling, sensitivity to light and sound. There are multitude of drugs available to treat migraine like triptans, non-steroidal anti-inflammatory drugs, ergots and opioids. But these drugs are associated with adverse effects especially triptans causing cardiovascular effects limiting its use. During last decade, calcitonin gene-related peptide (CGRP) has emerged as a possible mechanism for management of migraine. CGRP has been shown to release during episode of migraine attack and it may play a causative role in induction of migraine. Rimegepant is a novel CGRP antagonist has been approved by FDA for treatment of acute migraine. Rimegepant is a first oral CGRP antagonist compared to other gepants. The oral bioavailability of Rimegepant is $64 \%$ and high fat meal can decrease the Cmax, Tmax and area under the curve. This drug is mainly metabolized by CYP3A4 and to lesser extent by CYP2C9. Most common adverse reactions associated with this drug were nausea and urinary tract infection. Clinical trials for Rimegepant have been positive, and results suggest that the drug may be a new safe and effective option for treatment of acute migraine.
\end{abstract}

Keywords: Calcitonin gene-related peptide, Migraine, Rimegepant, Triptans

\section{INTRODUCTION}

Migraine is the most common neurological disorder presenting with the transient nature of primary headaches. ${ }^{1}$ Currently, many drugs are available in the market to treat migraine, such as triptans, non-steroidal anti-inflammatory drugs (NSAIDs), acetaminophen, ergots, and opioids. The main disadvantages of these drugs were either they have more contraindications or adverse effects. Previously, serotonin 5-HT1B and 5HT1D receptor agonists (triptans) were most commonly used for acute treatment of migraine. However, only $34 \%$ patients using triptans shows a good response while 30$40 \%$ patients have recurrent episodes. ${ }^{2,3}$ In addition, more than $50 \%$ patients who received triptans developed mild and severe adverse effects including cardiovascular effects, due to the vasoconstriction effect leading to fatal consequences and limit the use of triptans in patients with cardiovascular disease comorbidity. ${ }^{4}$ Most of the patients discontinued these drugs due to the lack of efficacy or troubling adverse effects. ${ }^{5}$ Thus, there is still an urgent need for the development of new therapeutic methods for acute management of migraine headache. 


\section{CALCITONIN GENE RELATED PEPTIDE AND MIGRAINE}

Calcitonin gene related peptide (CGRP) is a 37 amino acid long peptide produced by the neurons in central and peripheral nervous system. CGRP was found to be involved in various activities like neuroimmune activity, vasodilatation, bone metabolism and fracture healing. ${ }^{6}$ CGRP has been discovered to be associated with pathogenesis of migraine. During migraine attacks, it has been observed that there is an elevated levels of CGRP. ${ }^{7}$ there are possible mechanisms including central neurogenic vasodilation, inhibiting both vascular nociceptive transmission, and thalamic trigeminal nociceptive activation. ${ }^{8}$ There are several CGRP receptor antagonists (called gepants), including Olcegepant (BIBN4096BS), Telcagepant (MK-0974), (MK-3207), (BI-44370 TA), Rimegepant (BMS-927711), and Ubrogepant (MK-1602). ${ }^{9}$ According to previous studies, Olcegepant and BI-44370 have good efficacy against migraine but come with relatively high toxicity. These two types of gepants thus have limited clinical usefulness. ${ }^{10}$

Rimegepant is an oral CGRP antagonist approved by FDA on February 27,2020 for the acute treatment of migraine. While several parenteral antagonists of CGRP and its receptor have been approved for migraine therapy (e.g. erenumab, fremanezumab, galcanezumab), rimegepant and ubrogepant are the only members of the "gepants" family of medications remaining in development, and the only CGRP antagonists that possess good oral bioavailability. Antagonism of the CGRP pathway has become an attractive target for migraine therapy as, unlike the triptans, oral CGRP antagonists have no observed vasoconstrictive properties and are therefore safer for use in patients with contraindications to standard therapy. ${ }^{9}$

\section{MECHANISM OF ACTION}

The currently accepted theory of migraine pathophysiology considers dysfunction of the central nervous system, in particular the trigeminal ganglion, to be the root cause behind the condition. ${ }^{9}$ Stimulation of the trigeminal ganglion triggers the activation of trigeminal afferents that project to the spinal cord and synapse on various pain-sensing intra- and extracranial structures, such as the dura mater. Pain signals are then further transmitted via second-order ascending neurons to the brainstem, hypothalamus, and thalamic nuclei, and from there to several cortical regions (e.g. auditory, visual, motor cortices). ${ }^{9}$

The trigeminal ganglion appears to amplify and perpetuate the migraine headache pain through the activation of perivascular fibres and the release of molecules involved in pain generation, such as CGRP. ${ }^{9}$
The $\alpha$-isoform of CGRP, expressed in primary sensory neurons, is a potent vasodilator and has been implicated in migraine pathogenesis - CGRP levels are acutely elevated during migraine attacks, return to normal following treatment with triptan medications, and intravenous infusions of CGRP have been shown to trigger migrainelike headaches in migraine patients. In addition to its vasodilatory properties, CGRP appears to be a pronociceptive factor that modulates neuronal excitability to facilitate pain responses. ${ }^{11}$

Rimegepant is an antagonist of the calcitonin gene-related peptide receptor- it competes with CGRP for occupancy at these receptors, preventing the actions of CGRP and its ability to amplify and perpetuate migraine headache pain, ultimately terminating the headache. ${ }^{11}$

\section{CLINICAL PHARMACOLOGY}

\section{Pharmacodynamics}

Pharmacodynamics and clinical effects of Rimegepant are quite different and mechanism of action of the drug is still unknown. Even at a dose four times higher than recommended, it had no effect on QT interval.

\section{Pharmacokinetics}

Following oral administration, maximum concentration of the drug occurred at 1.5 hours. The absolute oral bioavailability is observed as approximately $64 \% .^{12}$

Experiments with high fat meal showed that Tmax is delayed by one hour, reduction in Cmax by 42 to $53 \%$ and a reduction of 32 to $38 \%$ in AUC. However, interaction of Rimegepant with various foods is yet to be studied. With plasma protein binding of $96 \%$, steady state volume distribution of the drug is estimated as $120 \mathrm{~L}$.

Mostly, the drug is metabolised by CYP3A4 and to a lesser extent by CYP2C9. The drug is mostly eliminated in unchanged form $(77 \%)$ with elimination half-life of 11 hours. The drug is seen to be eliminated mostly in faeces $(78 \%)$ and to a lesser extent in urine (24\%). Pharmacokinetics of the drug was independent of age, sex, race/ethnicity, body weight, or CYP2C9 genotype.

In patients with moderate renal impairment, $\mathrm{Cmax}$ and AUC were seen to be $40 \%$ higher than that of normal subjects. In patients of severe hepatic impairment, Cmax and AUC were twice than that of normal subjects.

\section{Drug interaction}

The drug is a substrate of CYP3A4 and CYP2C9 and not an inhibitor of CYP1A2, 2B6, 2C9, 2C19, 2D6 and UGT1A1 at clinically relevant concentrations. However, weak inhibition was seen with CYP3A4. Concomitant administration of strong inhibitors of CYP3A4 with Rimegepant should be avoided as they would result in 
increased exposure of the drug. However, with moderate inhibitors of CYP3A4, dose repetition should be avoided for 48 hours. On the contrary, simultaneous administration with moderate and severe inducers of CYP3A4, would result in decreased exposure of the drug affecting its efficacy. On co-administering with oral contraceptives (norelgestromin, ethinyl estradiol), midazolam or sumatriptan, no significant pharmacokinetic interactions were observed. As the drug is a substrate of P-gp and BRCP, effects of concomitant administration with these substrate inhibitors needs to be evaluated.

\section{Pharmacogenomics}

CYP2C9 activity is reduced in individuals with the CYP2C9*2 and CYP2C9*3 genetic variants. Cmax and AUC0-inf were similar in CYP2C9 metabolizers (normal and intermediate). However, no data is available regarding interaction with poor metabolizers.

\section{Special conditions}

Animal studies suggested decreased foetal body weight and an increased incidence of foetal variations on administering Rimegepant. Few studies suggested the increased occurrence of gestational hypertension and preeclampsia in pregnant females with migraine. No data is available assessing the effects of Rimegepant on birth defects in humans. Hence care should be taken while administering the drug in this group of patients. Efficacy of the drug is not established in paediatric population. In geriatric age group, pharmacokinetics of the drug was similar to that of younger age group. In patients with severe hepatic and renal impairment, the drug should not be administered.

\section{CLINICAL TRIALS}

\section{Clinical trials conducted to assess the efficacy of Rimegepant}

The efficacy and safety of Rimegepant in migraine is derived from phase 2 and phase 3 trials, which is summarized in Table $1.5,12,13$

\section{Safety and adverse effects}

The safety of Rimegepant was evaluated in two phase 3 clinical trials. In one of the phase 3 trial (NCT03461757), the most common on-study adverse events were nausea (11 [2\%] of 682 participants vs three [<1\%] of 693 participants) and urinary tract infection (ten [1\%] of 682 participants vs four [1\%] of 693 participants).

Table 1: Summary of clinical trials conducted to assess the efficacy of Rimegepant.

\begin{tabular}{|c|c|c|c|c|}
\hline Study & Study design & $\mathbf{n}^{\mathbf{a}}$ & Drugs compared & Outcome $^{b}$ ( 2 h pain freedom) \\
\hline Phase $2^{c}$ & $\begin{array}{l}\text { Double blind placebo } \\
\text { controlled } \\
\text { dose ranging study }\end{array}$ & 885 & $\begin{array}{l}\text { Placebo } \\
\text { Rimegepant75mg } \\
\text { Rimegepant150mg } \\
\text { Rimegepant300mg } \\
\text { Rimegepant600mg } \\
\text { Sumatriptan }\end{array}$ & $\begin{array}{l}15.3 \% \\
31.4 \% \\
32.9 \% \\
29.7 \% \\
24.4 \% \\
35 \%\end{array}$ \\
\hline Phase $3^{d}$ & $\begin{array}{l}\text { Double blind } \\
\text { Placebo controlled } \\
\text { Multicentric }\end{array}$ & 1466 & $\begin{array}{l}\text { Placebo } \\
\text { Rimegepant } 75 \mathrm{mg}\end{array}$ & $\begin{array}{l}11 \% \\
21 \%\end{array}$ \\
\hline Phase $3^{\mathrm{e}}$ & $\begin{array}{l}\text { Double blind Placebo } \\
\text { controlled Multicentric }\end{array}$ & 1186 & $\begin{array}{l}\text { Placebo } \\
\text { Rimegepant } 75 \mathrm{mg}\end{array}$ & $\begin{array}{l}12 \% \\
19.6 \%\end{array}$ \\
\hline
\end{tabular}

a - number of patients, $b-\%$ of patients achieving this outcome, c- NCT01430442

d- NCT03461757, e- NCT03237845

Table 2: Adverse events with Rimegepant 75 mg and placebo. ${ }^{5}$

\begin{tabular}{|lll|}
\hline Adverse events & Rimegepant $75 \mathbf{~ m g}(\mathbf{n = 5 4 3})$ & Placebo $(\mathbf{n = 5 4 3})$ \\
\hline & $\mathbf{N}(\mathbf{\%})$ & $\mathbf{N}(\mathbf{\%})$ \\
\hline Any adverse event & $93(17.1)$ & $77(14.2)$ \\
\hline Nausea & $10(1.8)$ & $6(1.1)$ \\
\hline Urinary tract infection & $8(1.5)$ & $6(1.1)$ \\
\hline Serious adverse event & $1(0.2)$ & $2(0.4)$ \\
\hline Serum AST/ALT above ULN & $13(2.4)$ & $12(2.2)$ \\
\hline Serum AST/ALT $>\mathbf{3}$ X ULN & 0 & 0 \\
\hline Total bilirubin $>\mathbf{2}$ x ULN & 0 & 0 \\
\hline
\end{tabular}

$\mathrm{a}=$ reported from NCT03237845, b - The serious adverse event reported in the Rimegepant group was back pain, and the serious adverse events reported in the placebo group were chest pain (1 patient) and urinary tract infection (1 patient). 
No serious adverse events were reported in treated participants. In other phase trial (NCT03237845), The most common adverse events were nausea $(1.8 \%$ in the Rimegepant group and $1.1 \%$ in the placebo group) and urinary tract infection ( $1.5 \%$ and $1.1 \%$, respectively). Serious adverse events were reported in one patient in the Rimegepant group (back pain) and in two patients in the placebo group (Table 2$)^{5}$

\section{CONCLUSION}

CGRP antagonists are greatest advances in the field of migraine. Rimegepant, one such drug can be used in treating acute migraine when triptans are not effective or contraindicated due to cardiovascular disorders. The monoclonal antibodies against CGRP and its receptor have high responder rates with favourable adverse event profiles. Given that approximately $40-50 \%$ of migraine patients do not respond to monoclonal antibodies, future studies should focus on identification of biomarkers that can predict treatment response. Collectively, novel migraine therapies represent a major progress in migraine treatment and will undoubtedly transform headache medicine.

\section{Funding: No funding sources Conflict of interest: None declared Ethical approval: Not required}

\section{REFERENCES}

1. Reuter U. GBD 2016: still no improvement in the burden of migraine. Lancet Neurol. 2018;17(11):92930.

2. Perry CM, Markham A. Sumatriptan. Drugs. 1998;55(6):889-922.

3. Géraud G, Keywood C, Senard JM. Migraine headache recurrence: relationship to clinical, pharmacological, and pharmacokinetic properties of triptans. Headache J Head Face Pain. 2003;43(4):376-88.

4. Derry CJ, Derry S, Moore RA. Sumatriptan (oral route of administration) for acute migraine attacks in adults. Cochrane Database Syst Rev. 2012;(2).
5. Lipton RB, Croop R, Stock EG, Stock DA, Morris BA, Frost M, et al. Rimegepant, an oral calcitonin gene-related peptide receptor antagonist, for migraine. N Engl J Med. 2019;381(2):142-9.

6. Naot D, Cornish J. The role of peptides and receptors of the calcitonin family in the regulation of bone metabolism. Bone. 2008;43(5):813-8.

7. Edvinsson L. Rimegepant oral disintegrating tablet for migraine. Lancet Lond Engl. 2019;394(10200):711.

8. Storer RJ, Akerman S, Goadsby PJ. Calcitonin generelated peptide (CGRP) modulates nociceptive trigeminovascular transmission in the cat. $\mathrm{Br} \mathrm{J}$ Pharmacol. 2004;142(7):1171-81.

9. Negro A, Martelletti P. Gepants for the treatment of migraine. Expert Opin Investig Drugs. 2019;28(6):555-67.

10. Xu F, Sun W. Network meta-analysis of calcitonin gene-related peptide (CGRP) receptor antagonists for the acute treatment of migraine. Front Pharmacol. 2019;10:795.

11. Martelletti P, Giamberardino MA. Advances in orally administered pharmacotherapy for the treatment of migraine. Expert Opin Pharmacother. 2019;20(2):209-18.

12. Marcus R, Goadsby PJ, Dodick D, Stock D, Manos G, Fischer TZ. BMS-927711 for the acute treatment of migraine: A double-blind, randomized, placebo controlled, dose-ranging trial. Cephalalgia. 2014;34(2):114-25.

13. Croop R, Goadsby PJ, Stock DA, Conway CM, Forshaw M, Stock EG, et al. Efficacy, safety, and tolerability of rimegepant orally disintegrating tablet for the acute treatment of migraine: a randomised, phase 3, double-blind, placebo-controlled trial. Lancet. 2019;394(10200):737-45.

Cite this article as: Ramasubbu SK, Dakshinamurthy S, Palepu S, Bandyopadhyay A, Sathish Kumar Rajendran SK. Rimegepant: first novel oral calcitonin gene-related peptide inhibitor for migraine. Int $\mathrm{J}$ Basic Clin Pharmacol 2020;9:829-32. 